\title{
How does leptin restore euglycemia in insulin-deficient diabetes?
}

\author{
Douglas Oberlin and Christoph Buettner
}

Department of Medicine and Diabetes, Obesity, and Metabolism Institute, Icahn School of Medicine at Mount Sinai, New York, New York, USA

\begin{abstract}
Insulin replacement is the cornerstone of type 1 diabetes (T1D) treatment; however, glycemic control remains a challenge. Leptin has been shown to effectively restore euglycemia in rodent models of T1D; however, the mechanism or mechanisms by which leptin exerts glycemic control are unclear. In this issue of the $J C I$, Perry and colleagues provide evidence that suppression of lipolysis is a key facet of leptin-mediated restoration of euglycemia. However, more work remains to be done to fully understand the antidiabetic mechanisms of leptin.
\end{abstract}

\section{Leptin for type 1 diabetes?}

Insulin has long been the therapeutic sine qua non for type 1 diabetes (T1D), where insulin deficiency is the hallmark of the disease. Insulin therapy comes with the risk of inadvertent hypoglycemia, which is the main barrier to tight glycemic control (1). The demonstration that leptin can restore euglycemia without correcting insulin deficiency in streptozotocininduced (STZ-induced) rodent models of T1D and thereby replace insulin was baffling and exciting (2-4). One reason that leptin replacement is effective in this model of T1D is that, in STZ-induced T1D, plasma leptin levels are reduced by more than $80 \%(5,6)$. In humans with T1D treated with insulin, plasma leptin levels appear to only decrease by approximately $50 \%(7,8)$. It is unclear whether leptin restores euglycemia in humans with T1D, although a trial to evaluate the effects of leptin in T1D patients was completed in 2013 (https://clinicaltrials.gov/ct2/show/ NCT01268644); however, the results of this study have not been made public.

A recent study demonstrated that leptin delivery to the brain is sufficient to restore euglycemia in STZ-treated rats (5), highlighting a key role of the CNS in systemic glucose homeostasis. This work also suggested that this experimental paradigm would allow exploration of the mechanisms through which the brain controls glucose homeostasis. Indeed, within the CNS, GABA and POMC neurons have been shown to be critical for the ability of leptin to restore euglycemia in rodent $\mathrm{T} 1 \mathrm{D}$ models (9).

\section{How does leptin restore euglycemia?}

While these studies support the brain as the primary site of leptin-mediated restoration of euglycemia, the peripheral effectors remain unknown. Initially, it was proposed that leptin prevents hyperglucagonemia, a common feature of T1D that was thought to be the primary cause of the hyperglycemia observed in T1D. Indeed, glucagon is suppressed after leptin treatment in T1D models (6), and mice that lack glucagon receptors are protected from hyperglycemia following STZ-induced T1D, despite persistent, absolute insulin deficiency (3). In these mice, restoration of hepatic glucagon receptor expression rapidly increased fasting hyperglycemia $(3,4)$. However, if glucagon was indeed the main driver of hyperglycemia in human T1D, then pancreatectomized humans that lack insulin and have markedly reduced

Related Article: p. 657

Conflict of interest: The authors have declared that no conflict of interest exists

Reference information: / Clin Invest. 2017;127(2):450-453. https://doi.org/10.1172/JCI91880.

glucagon levels should be protected from hyperglycemia. While pancreatectomized patients may have lower rates of diabetic ketoacidosis (DKA) than patients with autoimmune T1D, they still suffer from fasting hyperglycemia (10). Further, amelioration of hyperglucagonemia in rodent T1D models by administration of either a glucagon-like peptide 1 receptor agonist or a glucagon-neutralizing antibody only corrected hyperketonemia without reversing the hyperglycemia (11). Therefore, the ability of leptin to suppress glucagon may contribute to the restoration of euglycemia in T1D, but glucagon suppression does not appear to be the major mechanism through which leptin functions.

An alternative model recently proposed by Perry et al. suggests that leptin normalizes fasting blood glucose concentrations in T1D by suppressing the elevated hypothalamic-pituitary-adrenal (HPA) axis activity known to exist in T1D (Figure 1) $(6,12)$. In their 2014 study, Perry et al. demonstrated that glucocorticoids (GCs) are elevated and that lipolysis and gluconeogenesis are increased during hyperglycemia in a rat model of STZ-induced T1D. Moreover, i.v. administration of leptin reversed these STZ-induced effects within 6 hours of treatment, supporting HPA involvement in leptin-mediated actions (6). Perry and colleagues further suggested that excess delivery of nonesterified fatty acids (NEFAs) and glycerol to the liver increase hepatic acetylCoA concentrations and hepatic glucose production (HGP) and that these are key mechanisms driving hyperglycemia. Therefore, the ability of leptin to lower GCs and lipolytic flux restores euglycemia (6). This is an attractive model, as it provides substrate flux mechanisms that account for the hyperglycemia in T1D and implies that antagonizing GCs could represent an alternative therapeutic strategy for treating T1D. There are three caveats to this initial study, however. First, HGP was only studied after euglycemia had been 


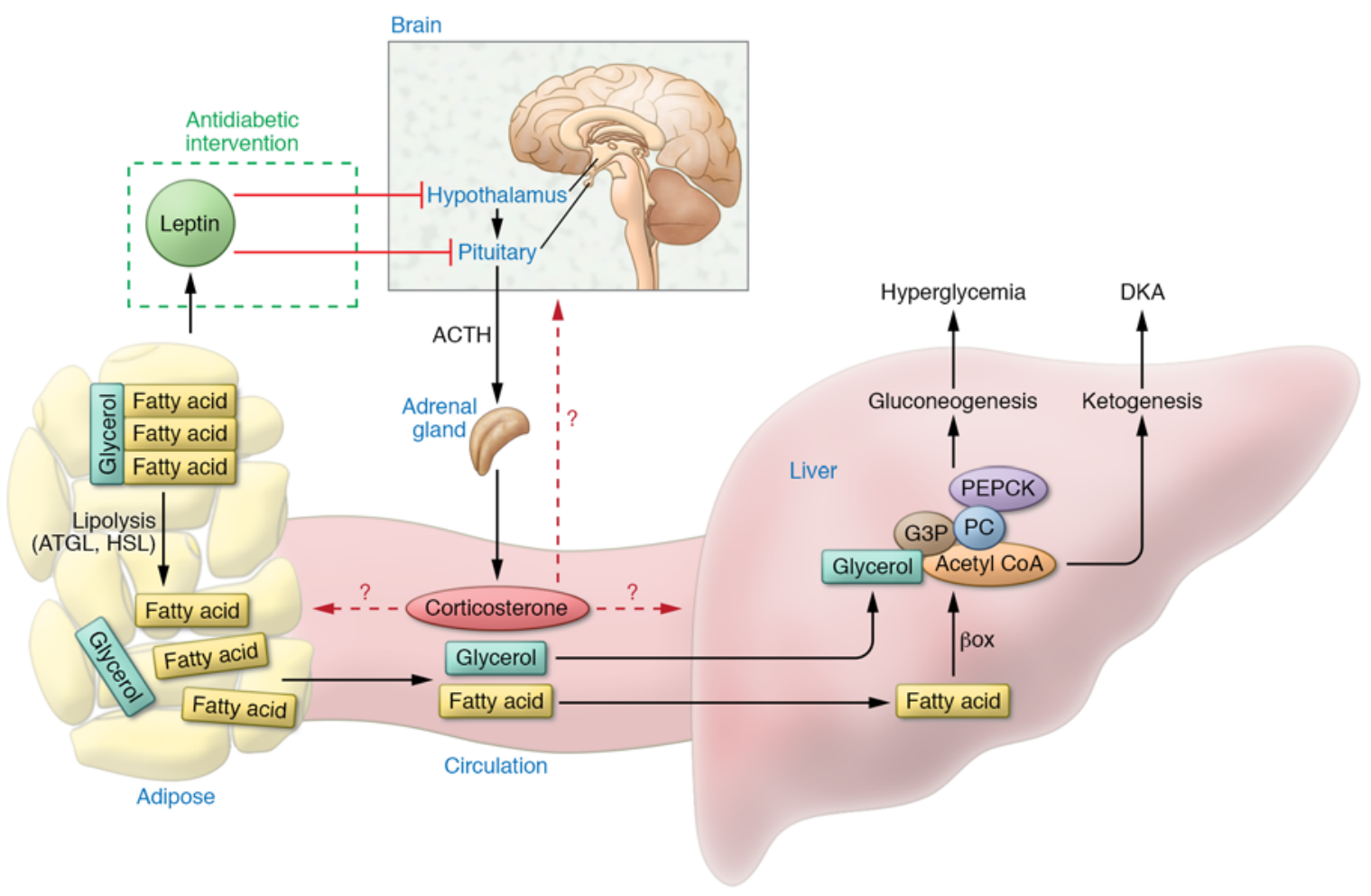

Figure 1. Model proposed by Perry et al. in this issue to explain the antidiabetic effects of leptin. Leptin restrains the activity of the HPA axis in T1D, reducing circulating corticosterone, which lowers lipolysis in adipose tissue. As of yet it remains unclear in what tissue and cell type GCs exert these lipolytic effects. Restrained lipolysis results in lower NEFAs and glycerol delivery to the liver, reducing gluconeogenesis and ketogenesis and thereby reversing hyperglycemia and DKA. PEPCK, phosphoenolpyruvate carboxykinase; G3P, glyceraldehyde 3-phosphate; HSL, hormone-sensitive lipase; PC, pyruvate carboxylase; $\beta$ ox, $\beta$ oxidation.

restored, and as hyperglycemia per se can increase HGP, it remains unclear whether the suppression of HGP is a direct effect of leptin or an indirect effect that is secondary to the restoration of euglycemia (13). Second, the role of enhanced lipolysis was modeled through an intralipid infusion that resulted in elevated fatty acid delivery to tissues such as the liver; however, this infusion also represents a caloric load that was not controlled for. Therefore, it remains unclear whether observed effects are due to elevated fatty acids or due to increased caloric load. Third, the study did not directly test the role of lipolysis in driving hyperglycemia.

Morton et al. questioned the HPA axis model and showed that i.c.v. infusion of leptin restored euglycemia even when the normalization of hypercortisolism in STZ-induced T1D rats was prevented (5). Further, inhibition of GC signaling either through adrenalectomy or pharmacological GC receptor blockade failed to normalize glycemia in T1D rats, suggesting that GC suppression alone is not sufficient to restore euglycemia. Morton and colleagues also showed that systemic administration of corticosterone at a dose that maintained elevated plasma GCs at a level characteristic of T1D did not reduce the glucose-lowering effect of continuous i.c.v. leptin infusion (5).

In this issue, a new study by Perry et al. follows up their initial work and demonstrates that leptin-mediated suppression of lipolysis is a key mechanism for the antidiabetic effects of leptin in rats with T1D (14). In contrast to the findings by Morton et al. (5), the current study found that normalization of hypercortisolism prevented restoration of glycemic control in i.v. leptin-treated T1D rats. Additionally, reduction of lipolysis with the adipose triglyceride lipase (ATGL) inhibitor ATGListatin rescued the ability of leptin to restore euglycemia in the setting of hypercortisolism. Similar to GCs, the infusion of acetate, which raises hepatic acetyl-CoA concentrations, prevented the antidiabetic action of leptin. Finally, within hours after adrenalectomy, euglycemia was restored in STZ-induced T1D rats, but not if corticosterone levels were maintained.
It remains puzzling that GCs have such different effects on the antidiabetic effects of leptin in these two studies. Perry et al. (14) argue that the studies by Morton and colleagues (5) were performed only 3 hours after food removal and that the postprandial state of the animals may have contributed to an inability to detect any effects of acute leptin treatment on plasma glucose concentrations. However, Schwartz pointed out in an e-letter that 16-hour fasts were used for their experiments (5). Additionally, several studies have demonstrated that leptin does restore euglycemia in both the fed and fasted state for an extended amount of time (i.e., for weeks; refs. 15-18). Hence, the task ahead is to understand how brain leptin is able to provide long-term restoration of euglycemia. Therefore, the ultimate validation of any such leptin-dependent mechanism will be to test whether leptin truly restores euglycemia for weeks, not just hours.

\section{Reconciling different models}

How can we resolve these divergent findings? Perry et al. point out that in the study 
by Morton et al., there was a moderate $\beta$ cell reserve, while there was none in their own study (14). Even very low insulin levels may prevent evaluation of insulin-independent effects of leptin, as the remaining insulin would be sufficient to suppress lipolysis such that DKA does not develop. As Morton et al. did not assess plasma ketones, it is possible that a fundamental difference is that one group is studying DKA, while the other is not; however, this then limits the relevance of the mechanisms proposed by Perry et al. to hyperglycemia in the setting of DKA. It remains questionable whether or not these effects are relevant to the chronic antidiabetic effects of leptin that Morton et al. and most other studies have evaluated. It is likely that animals in other studies, in which leptin was shown to restore euglycemia, also had minimal $\beta$ cell reserves, and in these cases, leptin restored euglycemia in the presence or absence of DKA (15-18).

Both groups argue that the other's animals experienced higher stress levels than animals in their own study. Morton et al. point out that the rats in the initial Perry et al. study received a higher dose of the STZ than their animals (5). In their current work, Perry et al. argue that the markedly higher adrenocorticotropic hormone (ACTH) levels observed by Morton et al. are an indicator of increased stress (14). While stress in physiological studies can markedly alter glucose homeostasis and metabolic control, it is difficult to judge whether there were indeed differences in animal stress levels in these two studies, and it remains to be determined why ACTH levels were so different. The groups used different strains of rats, which could also account for the discrepancy. Further, Morton et al. administered leptin via i.c.v. infusion in most of their studies, while in the Perry study, rats only received leptin via i.v. infusion. It is possible that i.c.v. leptin does not reach the same brain regions that i.v. leptin reaches and/or that i.c.v. leptin bypasses areas of reduced leptin transport that restrict access of i.v.-administered leptin to certain brain regions.

It should be noted that it has been reported to take two to three days for leptin to restore eyglycemia in STZ-induced T1D models in all previous studies, including that by Morton and colleagues $(15,16,18)$. The two studies by Perry et al. are the only reports that we are aware of where i.v. infusion of leptin acutely, within hours, restores euglycemia. It is unclear why this has not been observed in other studies, and this may point toward toxic effects of STZ in addition to the $\beta$ cell toxicity. Other studies usually started leptin treatment several days after STZ treatment and not within 24 hours, as done by Perry and Morton. This delay between STZ treatment and the experimental intervention does ascertain that STZ has been washed out and that no toxic STZ-specific effects linger. During this time, rats could be treated with low-dose insulin to prevent mortality from DKA.

Future studies should include a control group of STZ-induced T1D animals treated with a sodium/glucose cotransporter 2 (SGLT2) inhibitor, as an additional control group, which would normalize glycemia and prevent glucose toxicity despite insulin deficiency. This would allow to examine if leptin indeed directly suppresses HGP and/or lipolysis or weather the leptins ability to restrain HGP is simply a consequence of the amelioration of hyperglycemia through other mechanisms, given that hyperglycemia increases HGP.

It is not clear how GCs drive lipolysis, although they are commonly described as prolipolytic in vivo. GC receptors are expressed in many tissues including the brain and are permissive to myriad physiological processes (19). It might be assumed that GCs drive lipolysis by primarily by acting upon adipocytes, leading to increased gluconeogenesis and ketogenesis in the liver. Whether GCs acutely induce lipolysis in adipocytes directly, as shown in vivo by Perry and colleagues, remains controversial, as other recent studies show that even when exposed to high doses of GCs, adipocytes do not notably increase lipolysis during the first 16 hours (19-24). Hence, GCs may increase lipolysis in adipose tissue by acting on cell types other than adipocytes including those of the CNS. Further, GCs may impair central leptin signaling, similarly to high-fat feeding; however, if GCs prevent antidiabetic leptin action, it is unknown which tissues that express GC receptors are necessary for these effects. An even more important question is whether mice that lack GC receptors are chronically protected from hyperglycemia resulting from insulin deficiency.
A key tenet of the new study by Perry et al. is that a reduction of lipolysis prevents fasting hyperglycemia in T1D, although this study evaluated whether leptin plus GCs plus ATGListatin restored euglycemia, not whether ATGListatin solely, in the absence of leptin, restored euglycemia (14). The latter experiment would have been a more definitive approach to probing the role of lipolysis in T1D-associated hyperglycemia. An important confirmation of the model proposed by Perry et al. would be the demonstration that the inhibition of lipolysis indeed chronically ameliorates hyperglycemia. A study more than two decades ago examined the role of fatty acid flux to the liver in driving the hyperglycemia of T1D in STZ-induced rats using the lipolytic inhibitor acipimox (25). After eight days of acipimox treatment, plasma NEFAs were markedly lower, but fasting hyperglycemia was not reduced (24). Granted, the antilipolytic mode of action of acipimox and ATGListain differ substantially, as acipimox inhibits lipolysis by activating GPR109a and ATGListatin is a specific inhibitor of ATGL, which initiates lipolysis in adipose tissue. Hence, different mode of action of these lipolytic inhibitors may account for the varied efficacy in ameliorating fasting hyperglycemia in T1D. Whether prolonged treatment with ATGListatin is effective in reducing hyperglycemia in a T1D model has yet to be determined, but if it does, it may point to novel strategies to treat insulin-deficient diabetes beyond insulin.

\section{Acknowledgments}

This work was supported by NIH grants R01 AA023416 and DK082724 and the American Diabetes Association.

Address correspondence to: Christoph Buettner, Department of Medicine, Icahn School of Medicine at Mount Sinai, One Gustave L Levy Place, New York, New York 10029, USA. Phone: 212.241.3425; E-mail: christoph.buettner@mssm.edu.

1. Cryer PE. The barrier of hypoglycemia in diabetes. Diabetes. 2008;57(12):3169-3176.

2. Chinookoswong N, Wang JL, Shi ZQ. Leptin restores euglycemia and normalizes glucose turnover in insulin-deficient diabetes in the rat. Diabetes. 1999;48(7):1487-1492.

3. Lee Y, Wang MY, Du XQ, Charron MJ, Unger RH. Glucagon receptor knockout prevents insulindeficient type 1 diabetes in mice. Diabetes. 
2011;60(2):391-397.

4. Lee Y, et al. Metabolic manifestations of insulin deficiency do not occur without glucagon action. Proc Natl Acad Sci U S A. 2012;109(37):14972-14976.

5. Morton GJ, Meek TH, Matsen ME, Schwartz MW. Evidence against hypothalamic-pituitary-adrenal axis suppression in the antidiabetic action of leptin. J Clin Invest. 2015;125(12):4587-4591.

6. Perry RJ, et al. Leptin reverses diabetes by suppression of the hypothalamic-pituitary-adrenal axis. Nat Med. 2014;20(7):759-763.

7. Hanaki K, Becker DJ, Arslanian SA. Leptin before and after insulin therapy in children with new-onset type 1 diabetes. J Clin Endocrinol Metab. 1999;84(5):1524-1526.

8. Hathout EH, Sharkey J, Racine M, Ahn D, Mace JW, Saad MF. Changes in plasma leptin during the treatment of diabetic ketoacidosis. J Clin Endocrinol Metab. 1999;84(12):4545-4548.

9. Xu Y, Chang JT, Myers MG, Xu Y, Tong Q. Euglycemia restoration by central leptin in type 1 diabetes requires stat 3 signaling but not fast-acting neurotransmitter release. Diabetes. 2016;65(4):1040-1049.

10. Barnes AJ, et al. Ketoacidosis in pancreatectomized man. N Engl JMed.1977;296(22):1250-1253.

11. Meek TH, et al. Evidence that in uncontrolled diabetes, hyperglucagonemia is required for ketosis but not for increased hepatic glucose production or hyperglycemia. Diabetes.
2015;64(7):2376-2387.

12. Chan O, Inouye K, Vranic M, Matthews SG. Hyperactivation of the hypothalamo-pituitaryadrenocortical axis in streptozotocin-diabetes is associated with reduced stress responsiveness and decreased pituitary and adrenal sensitivity. Endocrinology. 2002;143(5):1761-1768.

13. Rossetti L, Smith D, Shulman GI, Papachristou D, DeFronzo RA. Correction of hyperglycemia with phlorizin normalizes tissue sensitivity to insulin in diabetic rats. JClin Invest. 1987;79(5):1510-1515.

14. Perry RJ, et al. Mechanism for leptin's acute insulin-independent effect to reverse diabetic ketoacidosis. JClin Invest. 2017;127(2):657-669.

15. Hidaka S, et al. Chronic central leptin infusion restores hyperglycemia independent of food intake and insulin level in streptozotocin-induced diabetic rats. FASEB J. 2002;16(6):509-518.

16. Lin CY, Higginbotham DA, Judd RL, White BD. Central leptin increases insulin sensitivity in streptozotocin-induced diabetic rats. Am J Physiol Endocrinol Metab. 2002;282(5):E1084-E1091.

17. Yu X, Park BH, Wang MY, Wang ZV, Unger RH. Making insulin-deficient type 1 diabetic rodents thrive without insulin. Proc Natl Acad Sci US A. 2008;105(37):14070-14075.

18. Fujikawa T, Chuang JC, Sakata I, Ramadori G, Coppari R. Leptin therapy improves insulin-deficient type 1 diabetes by CNS-dependent mechanisms in mice. Proc Natl Acad Sci USA.
2010;107(40):17391-17396.

19. Lee MJ, Pramyothin P, Karastergiou K, Fried SK. Deconstructing the roles of glucocorticoids in adipose tissue biology and the development of central obesity. Biochim Biophys Acta. 2014;1842(3):473-481.

20. Lee MJ, Fried SK. Glucocorticoids antagonize tumor necrosis factor- $\alpha$-stimulated lipolysis and resistance to the antilipolytic effect of insulin in human adipocytes. Am J Physiol Endocrinol Metab. 2012;303(9):E1126-E1133.

21. Xu C, et al. Direct effect of glucocorticoids on lipolysis in adipocytes. Mol Endocrinol. 2009;23(8):1161-1170.

22. Samra JS, Clark ML, Humphreys SM, MacDonald IA, Bannister PA, Frayn KN. Effects of physiological hypercortisolemia on the regulation of lipolysis in subcutaneous adipose tissue. J Clin Endocrinol Metab. 1998;83(2):626-631.

23. Fain JN, Cheema P, Tichansky DS, Madan AK. Stimulation of human omental adipose tissue lipolysis by growth hormone plus dexamethasone. Mol Cell Endocrinol. 2008;295(1-2):101-105.

24. Slavin BG, Ong JM, Kern PA. Hormonal regulation of hormone-sensitive lipase activity and mRNA levels in isolated rat adipocytes. J Lipid Res. 1994;35(9):1535-1541.

25. Kim YW, Kim JY, Lee SK. Effects of phlorizin and acipimox on insulin resistance in STZ-diabetic rats. J Korean Med Sci. 1995;10(1):24-30. 